\title{
A catalog of the types of Hydraenidae (Staphylinoidea) and of Hydrophiloidea (Insecta, Coleoptera, Polyphaga) deposited at the Museo Argentino de Ciencias Naturales, Buenos Aires
}

\author{
Axel O. BACHMANN \\ Museo Argentino de Ciencias Naturales "Bernardino Rivadavia", Av. A. Gallardo 470, 1405 Buenos Aires, \\ Argentina. Researcher of the CONICET. e-mail bachmann@bg.fcen.uba.ar.
}

\begin{abstract}
The type specimens (all accepted categories) of the Hydraenidae (Staphylinoidea) and the families currently included in the superfamily Hydrophiloidea, deposited at the Museo Argentino de Ciencias Naturales are listed; 125 names are recorded ( 2 of Hydraenidae, 123 of Hydrophiloidea); 79 of them (63\%) are represented here by name-bearing types (holotypes, syntypes and lectotypes), Later combinations and/or current binomina are mentioned when known to the author. In order to facilitate future research, two lists are added: 1 . of specimens labelled as types of names not found in the literature, and presumably not published, and 2. of specimens labelled as types, but not originally included as such, and published or not after the original description.
\end{abstract}

Key words: Hydraenidae, Hydrophiloidea, type specimens

A list of the type specimens of species of Hydraenidae (Staphylinoidea) and of Hydrophiloidea, deposited at the Entomological Division of this Museum is here given; they correspond to 125 names, 2 of Hydraenidae, 123 of Hydrophiloidea: 50 holotypes, 52 syntypes (of 17 names; one of them dubious), 12 lectotypes, 722 paratypes (including 36 allotypes), and 41 paralectotypes; it follows that 79 of these 125 names $(63 \%)$ are here represented by name-bearing types ('primary' types).

Although the Helophoridae, Georissidae (neither group represented in this catalog), Epimetopidae, Hydrochidae and Hydrophilidae are now treated as separate families, they are here included in a single list.

Specific and subspecific names are alphabetically entered, as is usual in type specimen catalogs; each name is followed by the generic one (and subgeneric one if it was mentioned) as it was spelled in the original binomen, or by the generic and specific ones in the case of subspecies; the bibliographic citation follows, as well as an account of the types, if these were mentioned, starting with those deposited in this Museum. An account of the type specimens actually housed follows. According to the International Code of Zoological Nomenclature (4 $4^{\text {th }}$ edition, 1999), allotypes are not 'name bearing types'. If the country is not mentioned, Argentina is meant; in every other case, the country is mentioned first. Some specimens bear a registration number, entered in the Entomological Division register; numbers under 10000 correspond to the old ge neral register of the Museum. Later nomenclatural or taxionomical changes are added, if these are known by the author.

Entries with no description of the labels correspond to formally correct cases; in the remaining entries such a description helps to state the actual condition of the specimens mentioned.

For the identification of types not formally designated in the original publication (art. 72.4 of the Code), evidences from the labels were used. When a holotype, or its equivalent ("typus", «type» or "tipo") was not formally designated, and the amount of specimens examinated was not mentioned, it is assumed that there was a series of syntypes (recommendation $73 \mathrm{~F}$ of the Code), eventually 'single syntypes'.

In the collection are found some specimens labelled as types, of names not found in the literature, and assumed not to have been published. These names are probably not available in the sense of the Code; however, in order to assist in future research, they are separately listed.

Specimens designated as types after the original publication date, published or not, cannot be accepted as included in the type series; however, also in order to assist in future research, they are included in a third list, along with other specimens labelled as types, but not included in 
the original publication. In each case a 'Note' is added to clear their situation.

Institutions mentioned in the list as housing some types, are identified as follows: BM: The Natural History Museum, London, UK; CAS: California Academy of Sciences, San Francisco, USA; CEA: Centro de Ecología Aplicada del Litoral, Corrientes, Argentina; IML; Instituto y Fundación Miguel Lillo, Tucumán, Argentina; INPA: Instituto Nacional de Pesquisas da Amazônia, Manaus, Brasil; ISNB: Institut Royal des Sciences Naturelles de Belgique, Bruxelles, Belgique; MACN: Museo Argentino de Ciencias Naturales, Buenos Aires, Argentina; MG: Museu Emilio Goeldi, Belém, Brasil; MHNG: Musée d'Histoire Naturelle, Genève, Suisse; MLP: Museo de La Plata, La Plata, Argentina; MLS: Museo de Historia Natural La Salle, Caracas, Venezuela; MINHN: Muséum National d'Histoire Naturelle, Paris, France; MP: Museum Pragae, Praha, Czech Republic; MZ: Museu de Zoologia, São Paulo, Brasil; TM: Transvaal Museum, Pretoria, South African Republic; UBA: University of Buenos Aires [now in MACN]; USNM: United States National Museum (Smithsonian Institution), Washington, USA; ZMB: Zoologisches Museum, Berlin-Dahlem, Germany; ZMHI: Zoologisches Museum, Hamburg, Germany; ZSB: Zoologische Staatssammlung Bayerns, München, Germany. Personal collections are mentioned by the names of their owners.

The author is deeply indebted to Drs. Adriana Oliva and Liliana A. Fernández for their valuable assistance in former drafts of this paper.

\section{CATALOG OF THE TYPES}

\section{Hydraenidae}

anisonycha [Hydraena]. Perkins 1980: 37, 70, 191-193, f. 2 C,E, 50 B, 57 A-F, 58 B, 153 H: Holot. o', allot. $\$, 1714$ parat. Colombia: Cundinamarca, and Colombia: Meta, at USNM.

1 parat. on a card, Colombia: Cundinamarca.

francki [sub Francki] [Ochthebius]. Bruch 1915: 464-465, f. 18: 10 ex. Buenos Aires.

1 synt. on a card, B. Aires, ex coll. Bruch.

Note: Recorded under Ochthebius (Hymenodes) by Knisch (1924b: 23), and under Gymnochthebius by Perkins (1980: 398, 250, 264-265, f. 86 C-E, 177).

\section{Hydrophiloidea}

acaragua [Tropisternus (Pristoternus)]. Bachmann 1970: 58-59, f. 1-4: Holot. $\delta$, allot. $\%, 2$ parat. Misiones Prov.: Oberá: Barra Bonita; 1 \& parat. Misiones Prov: Concepción Dept.: S. María; 1 q parat.
Misiones Prov.: Bonpland, in coll. Bruch. Other 2 parat. at MLP, 5 parat. in coll. Bachmann.

Holot. O pinned, with a glass microvial, allot. , 4 parat. with glass microvials, all pinned, Misiones: Oberá: Barra Bonita, 2 of themex coll. Bachmann; 1 q parat. Misiones: Bonpland, ex coll. Bruch; 1 \& parat. Misiones: S. María, ex coll. Bachmann.

Note: Pristoternus was synonymized with Tropisternus (Tropisternus) by Hansen (1989:54), and revalidated as a subgenus of Tropisternus by Bachmann (1993: 50, 72).

acutidens [Hydrobiomorpha]. Bachmann 1988: 8, 34-35, f. 19 A-H: Holot. O' Brasil: M. Grosso State: Corumbá, ex coll. Pellerano.

Holot. O", pinned; Brasil: Mato Grosso: Corumbá, ex coll. Pellerano.

adocetus [Guyanobius]. Spangler 1986: 587-593, f. 1-24: Parat. Guyana: MazarunimPotaro District: Takutu Mountains. Holot. $\delta$, allot. and parat. idem at USNM, parat. at several institutions.

$1 \delta, 1 \%$ parat. on pointed cards, Guyana: Mazaruni-Potaro District: Takutu Mountains

aequatorialis [Hemiosus]. Oliva 1994a: 276, 283284 , f. 11, 21, 30: 1 ơ 3 \% parat. Venezuela: Táchira State: Santo Domingo. Holot. and allot. idem at BM.

10 , 3 o parat. on cards, the $\delta$ with dissected parts glued to the same card, and with a plastic microvial, Venezuela: Táchira: Santo Domingo.

apicalis [Hemiosus]. Oliva 1994a: 280, 286-288, f. 1, 7, 13, 23, 31.: Holot. $\delta$, allot. $\$, 5$ Jै, 4 \% parat. Misiones Prov: Bernardo de Irigoyen: Uruguá-1 River; 6 6, 9 \% parat. Uruguay: Lavalleja Dept.: Minas. Other parat. at BM, MHNG, MZ [now at INPA], USNM.

Holot. $\delta$, allot. $\%, 11$ o, 13 \& parat. on cards, the holot. and 70 parat. with dissected parts glued to the same cards, holot. and 13 parat. with plastic microvials, Misiones: B. de Irigoyen: Uruguá-\{ River; 60,9 parat. on cards, 6 of them with dissected parts glued to the same cards, 6 of them with a plastic microvial, Uruguay: Lavalleja: Minas: El Perdido creek.

arcanus [Berosus]. Knisch 1924a: 137-139: 4 ex. (the author was allowed to retain one of them), Bolivia: Cuatro Ojos, leg. Lizer and Déletang, sent by Bruch.

3 paralectot, on cards, Bolivia: Cuatro Ojos, labelled "Kniždet. / Berosus [on 1]/ arcanus [on all]" handwritten by Knisch, ex coll. Lizer y Trelles.

Note: Lectot. and paralectot. designated by Oliva (1995b: 77).

argentinense [Phaenonotum]. Bruch 1915: 465466, f, 19: Several ex. among rotted roots of ombú 
[Phytolacca dioica], and in an aged stem of ceibo [Erythrina crista-galli], B. Aires Prov: La Plata, and Río Santiago; $=P$ argentinum Régimbart in litt.

Lectot. $\%, 1$ o paralectot. on cards, B. Aires Prov., labelled "Typus" on pale green paper, the lectot. in addition "argentinum / Rég. n. sp." handwritten by Régimbart, ex coll. Bruch.

Notes: There are 2 paralectot. at MLP, fide Fernández \& Cabrera (1996: 3-4). Lectot. and paralectot. designated by Archangelsky (1991: $160)$.

argentinensis [Derallus]. Bruch 1915: 459-460, f. 14: Several ex. Buenos Aires Prov: La Plata; = Derallus argentinus Régimbart in litt.

Holot., 2 parat. on cards, B. Aires Prov., labelled "Typus" and "Cotypus" respectively, on pale green paper, the "Typus", in addition, "argentinus Rég. / n. sp. type" handwritten by Régimbart, ex coll. Bruch.

atratus [Helochares]. Bruch 1915: 451-452, 481, $\mathrm{f}$. 4: Several ex., Buenos Aires: La Plata and B. Aires. Name of Régimbart in litt.

Lectot. $\delta, 2$ paralectot. on cards, with glass microvials, B. Aires, labelled "Typus" on pale green paper, and "atratus Rég. / n. sp. type" handwritten by Régimbart, ex coll. Bruch.

Notes: There is 1 paralectot. at MLP, fide Fernández \& Cabrera (1996: 4). Recorded under Helochares (Helochares) by Knisch (1924b: 196). Lectot. $\delta$ and 2 paralectot. designated by Fernández (1982a: 35-36). There is a further ex. from Misiones, not mentioned in the original publication, labelled "Cotypus" by Bruch (see below under atratus in Specimens labelled as types but not included in the original series).

avernus [Berosus]. Oliva 1993: 93-94, f. 17-23:4 ठै, 2 \& parat. Brasil: S. Paulo State?: Monte Alegre Ido Sul?], Holot. $\delta^{*}$, allot. $\$, 5 \delta^{\circ}, 2$ q parat. at BM; $1 \delta^{\circ}$ at IML; 10,1 at MZ; 10 at USNM.

$4 \delta, 2 \%$ parat. on cards, Brasil: Monte Alegre, the 40 with plastic microvials.

bacchusi [Hemiosus]. Otiva 1994a: 282, 288289 , f. $14,24,31: 2$ đै, 3 o parat. Venezuela: Táchira State: Santo Domingo. Holot. $ठ$, allot. $9,20,5 \%$ parat. idem at BM. Other parat. idem at MZ [now at INPA], USNM, ZSM.

20,5 parat. on cards, 4 of them with dissected parts glued to the same cards, the $2 \delta$, in addition, with plastic microvials, Venezuela: Táchira: S. Domingo.

batesi [Berosus]. Oliva 1993: 88-91, f. 5-8: 3 ठ̊, 3 \% parat. Brasil: Amazonas State: Santarem; 10 parat. Brasil: Amazonas State: Villa Nova; 1 of parat. Brasil:
Amazonas State: Paraná da Terra Nova. Holot. $\delta^{2}$ Brasil: Santarém, allot. 9 from Brasil: Obidos, and parat., at BM; other parat. at INPA and ISNB.

3 \%, 3 \% parat., Brasil: Amazonas: Santarém; 28 parat. with plastic microvials, Brasil: Amazonas: Solimões, all on cards.

bolivianus [Helochares (Helochares)]. Fernández 1989a: 146, 148, f. 2, 3: Holot. Oै Bolivia: Santa Cruz Dept: Gutiérrez Prov: Nueva Moka.

Holot. $\delta$, pinned, with a glass microvial, Bolivia: S. Cruz: Gutiérrez: N. Moka.

brachyphallus [Derallus]. Oliva 1983b: 346, pl. i: f. 2 a-c, ii: f. 2: 1 \% parat. Brasil: Amazonas State: Moravia River. Holot. 0 , allot. $\$$ at INPA.

1. parat. on a card, Brasil:Amazonas: Tiriyos Reservation, Moravia River.

brasiliensis [Hydrobiomorpha]. Bachmann 1988: 9, 41-42, f. 26 A-1: Holot. Ő Brasil, ex coll. Bachmann.

Holot. $\delta$, pinned, with a glass microvial, "Brazils".

brevibasis [Berosus]. Oliva 1989: 81, 165-167, 198, f. 264-268: 1 \%, 1 \& parat. Brasil: Mato Grosso State. Holot. $\delta$, allot. $Q, 2 \delta$, 19 parat. at $B M$.

$1 \delta, 1$ q parat. on cards, Brasil: Mato Grosso.

breviusculus [Philhydrus]. Bruch 1915: 455-456, f. 10: Few ex. Buenos Aires Prov: La Plata, and Jujuy. Name of Régimbart in litt.

Lectot. , B. Aires Prov, labelled "Typus" on pale green paper, and "breviusculus / Rég. n. sp. type" handwritten by Régimbart; paralectot. \& Jujuy, labelled "Philhydrus / breviusculus" handwritten by Régimbart, both on cards, ex coll. Bruch.

Notes: Recorded under Enochrus (Lumetus) by Knisch (1924b: 207); under Enochrus (Hugoscottia) by Orchymont (1937: 254). Lectot. and paralectot. designated by Fernández (1989b: 123-124, f. 5-7).

bruchi [Hemiosus]. Knisch 1924a: 139-140: 8 ex. (3 in coll. Knisch) Córdoba Prov: Aita Gracia, in coll. Bruch.

5 synt. on cards, on 2 pins $(2+3)$, Córdoba: Alta Gracia, labelled "Typus" on pale green paper, and "Knisch det, 1923 / Hemiosus / Bruchi Knisch", handwritten by Knisch, ex coll. Bruch.

Note:There are 2 further ex. on cards, labelled "Cotypus", det. Knisch, ex coll. Bruch (see below under bruchi in Specimens labelled as types but not included in the original series).

bruchi [Hydrochus]. Knisch 1924a: 115-116: 2 ex. Neuquen: Zapala, in coll. Bruch (the author was allowed to retain one of them). 
1 synt, on a card, Neuquen, labelled "Typus" on pale green paper, and "Knisch det. 1923 / Hydrochus / Bruchi m.", ex coll. Bruch.

bruchianus [Berosus]. Knisch 1924a: 126-12: $8 \mathrm{ex}$. Buenos Aires, in coll. Bruch, 1 of them labelled "Berosus chalcocephalus Rég.".

Lectot. on a card, labelled "Typus" on pale green paper, and "Knisch det. 1922 / Berosus (Enoplurus) / Bruchianus m." handwritten by Knisch; 5 paralectot. on cards, on 4 pins, labelled "Cotypus" on pale green paper, and "Knisch det. 1922./Bruchianus m.", all B. Aires, ex coll. Bruch.

Note: Lectot. and paralectot. designated by Oliva (1985: 191-194) sub Berosus (Enoplurus) bruchianus $=$ B. valparadiseus Moroni 1973 .

burmeisteri [Tropisternus (Pristoternus)]. Fernández \& Bachmann 1981: 274-275, f. 12-16: Holot. ¿, allot. Entre Ríos Prov: Primero de Mayo; 1 parat. E. Ríos Prov: Paraná, ex coll. Burmeister; 4 parat. Santa Fe Prov: Cayastá; 3 parat. Buenos Aires Prov: Paraná River delta; 5 parat. Corrientes Prov.: San Cosme; 3 parat. Corrientes Prov.: Santo Tomé; 1 parat. Uruguay, and 1 parat. without locality label, the last 2 ex coll. Burmeister. Other parat. from the same and other localities at IML, MLP, and coll. Bachmann.

Holot. o', allot. $\$, 3$ parat., E. Ríos: Primero de Mayo; 1 parat. E. Ríos: Paraná, ex coll. Burmeister; 5 parat. E. Ríos: Lazo, ex coll. Bachmann; 3 parat. Corrientes: S. Cosme; 4 parat. Corrientes: S. Tomé, ex coll. Pellerano; 4 parat. B. Aires: Paraná River delta; 7 parat. S. Fe: Cayastá, 3 of them ex coll. Bachmann; 2 parat. S. Fe: Villa Ana, ex coll. Bachmann; 1 parat. "Banda Oriental" [Uruguay] ex coll. Burmeister; 1 parat. without locality label, ex coll. Burmeister. All pinned.

Notes: There are 5 parat. at MLP, fide Fernández \& Cabrera (1996: 4). Currently in Tropisternus (Tropisternus).

burruyacu [Epimetopus]. Oliva 1987: 4, 7, pl. i: f. 1, 2, 5, 6; pl. ii: f. 1, 2: Holot. $\delta^{*}, 5$ parat. Tucumán Prov: Burruyacu Dept., 2 of them Salas Stream, 3 of them La Cocha, Other parat. at BM, IML, MZ, USNM, and coll. Oliva.

5 parat. on cards, with plastic microvials, Tucumán: Burruyacu, 2 of them Salas Stream, 3 La Cocha: Pizarros Dam.

caratinga'[Hydrobiomorpha]. Bachmann 1988: 9, 35, f. 20 A-L: Holot. $\delta$, allot. $\subsetneq$ Brasil: Minas Gerais State: Caratinga.

Holot. ơ with a glass microvial, allot. 9 , Brasil: M. Gerais: Caratinga, both pinned.

caribense [Phaenonotum [(Phaenonotum)]. Archangelsky 1990: 218-219, f. 1-3: Holot. o, allot. $\$$, 11 parat. Venezuela: Barinas State: S. Bárbara.
Holot. 8 , allot., , both with plastic microvials; 8 parat., all on cards, Venezuela: Barinas: S. Bárbara.

chaquensis [Helochares (Helochares)]. Femández 1982b: 87, f. 1-3: 10 parat. Brasil: [Mato Grosso State]: Corumbá, ex coll. Pellerano. Holot. ó, allot. $\$, 10,1$ ? parat, Chaco Prov.: S. Bernardo, at MLP; parat. Formosa Prov. (1), and Chaco Prov. (1), in coll. Bachmann.

10 parat., partially dissected, parts glued to 2 cards, Formosa: Pilcomayo Nat. Park: Blanca Pond, ex coll. Bachmann.

circumcinctus [Philhydrus]. Bruch 1915: 454-455, f. 8: Several ex. Buenos Aires Prov: La Plata. Name of Régimbart in litt.

Lectot. $\$$ on a card, B. Aires, labelled "Typus" on pale green paper, and "Philhydrus / circumcinctus / Rég. typ." handwritten by Régimbart, ex coll. Bruch.

Notes: There is 1 paralectot. at MLP, fide Fernández \& Cabrera (1996: 4). Recorded under Enochrus (Lumetus) by Knisch (1924b: 207); under Enochrus (Methydrus), ando lectot. and paralectot. designated by Fernández (1989b: 126-127, f. 12-1.5)

cochlearis [Helochares (Helochares)]. Fernández 1982b: 89, f. 6: Holot. $\delta$, allot. $\&, 8$ parat. Corrientes Prov: Santo Tomé, ex coll. Pellerano; 5 parat. Paraguay: Caaguazú Dept.; 2 parat. Paraguay: S. Pedro Dept.: Carumbé. Other parat. at IML.

Holot. ठ with a glass microvial, allot. 9,8 parat., all on cards, the parat. on 4 pins, 2 of them with glass microvials, Corrientes: S. Tomé; 5 parat. on 4 pins, 3 of them with glass microvials, Paraguay: Caaguazú.

coelacanthus [Berosus]. Oliva 1989: 78, 99-101, 198, f. 82-88: Holot. ठ, allot. $\$, 5$ o', 5 \% parat. Catamarca: Rodeo. Other parat. at BM, MZ, USNM.

Holot. $\$$, allot. \&, 10 parat. on cards, holot., allot. and 1 parat. with plastic microvials, Catamarca: Rodeo.

coheni [Chaetarthria]. Spangler 1977: 566-573, f. 1-10: Parat. Ecuador: Guayas Prov.: Olon. Holot. $\delta$, allot., parat. idem at USNM, parat. at several institutions.

10 , 1 q parat. on pointed cards, Ecuador: Guayas Prov.: Olon.

confusa [Helobata]. Fernández \& Bachmann 1987: 155-156, f. 12-14: Holot. Ot, allot. \& Paraguay: Asunción; 10 parat. 27351 from Paraguay. 1 ơ parat. Entre Ríos Prov: Victoria Dept.: Manantiales Creek, and 3 parat. Paraguay: Concepción Dept.: Puerto Vallemí, in coll. Bachmann.

1 parat. 27351 on a card, with a glass microvial, Paraguay: Puerto Guaraní; 3 parat. 
Paraguay: P. Vallemí, 1 of them pinned and with a glass microvial, the remaining on cards, ex coll. Bachmann; 1 parat. in alcohol, with a glass microvial, E. Ríos: Victoria: Manantiales Creek.

cordobana [Anacaena]. Knisch 1924a: 122-123: 6 ex. Córdoba Prov.: Alta Gracia, coll. Bruch.

2 synt. (both?) on cards, Córdoba: Alta Gracia: La Granja, labelled "Typus" and "Cotypus" respectively, on pale green paper, ex coll. Bruch.

Note: The "Typus" was labelled by Knisch, but the "Cotypus" only by Bruch, suggesting that it might not be a type specimen.

corruscans [Hydrochus]. Bruch 1915: 462-463, f. 16: Buenos Aires Prov: La Plata, always very abundant. Name of Régimbart in litt.

11 synt. on cards, on 7 pins $(1+1+1+3+2$ [one ex. missing] $+1+2$ ), the first 3 labelled "Typus", the remaining 4 "Cotypus" on pale green paper, the first one labelled "Hydrochus / corruscans / Rég. typ" handwritten by Régimbart, cut. Two of them with plastic microvials, B. Aires.

Notes: There are 7 synt. at MLP, fide Fernández \& Cabrera (1996: 5). A synonym of Hydrochus obscurus Sharp 1882, fide Oliva (1992: $90,95-97$, f. 5,27 ).

corumbaensis [Helobata]. Fernández \& Bachmann 1987: 155, f. 8-11: Holot. ơ Brasil: Mato Grosso State: Corumbá, in coll. Bruch.

Holot. $\delta$ on a card, with a glass microvial, Brasil: Mato Grosso: Corumbá, ex coll. Bruch.

corumbana [Anacaena]. Orchymont 1938; 267 . Type and many parat. Brasil: Mato Grosso State: Corumbá.

2 parat. on cards, on 1 pin, Brasil: M. Grosso: Corumbá, labelled "Para- / type" on pink paper, and "A. d'Orchymont det. / Anacaena / corumbana m.", handwritten by Orchymont, ex coll. Bruch.

cossyphoides [Helopeltis]. Bruch 1915: 458-459, 483, f. 13: 8 ex. Buenos Aires Prov: La Plata.

2 paralectot. on cards, B. Aires Prov, labelled "Typus" and "Cotypus" respectively, on pale green paper, and "Helopeltis / cossyphoides / m." (on one, "Bruch" on the other) handwritten by Bruch, ex coll. Bruch.

Notes: Synonymized with Helobata striata (Brulle 1841) by Knisch (1924b: 223). Resurrected from synonymy, recorded as Helobata cossyphoides, and lectot: and 2 paralectot. designated by Fernández \& Bachmann (1987: 151-153, f. 2-4); lectot. at MLP, fide Fernández \& Cabrera (1996: 5).

costalis [Hemiosus]. Oliva 1994a: 282, 289-290, f. $2,8,15,25,31: 3$ o parat. Brasil: Amazonas State: lower Aripuaná Stream. Holot. $\delta$, allot. $\%$, parat. idem at $M Z$ [now at INPA], and ZSM.

30 parat. on cards, 2 of them with dissected parts glued to the same cards, all 3 with plastic microvials, Brasil: Amazonas: lower Aripuaná Stream.

costera [Hydrobiomorpha]. Bachmann 1988:9,2324, f. 10 A-G: Holot. O, 1 parat. O", without locality label (presumably Brasil: Rio de Janeiro).

Holot. $\delta$, ex coll. Burmeister, 1 o parat., both pinned and with glass microvials.

cristatus [Hydrochus]. Oliva 1996b: 307, 319-321, f. 15, 16: $2 \%$ parat. Brasil: Amazonas State, near Manaos. Holot. + Brasil: Pará State: Belém, at MZ.

2 \& parat. on cards, dissected parts glued to the same cards, Brasil: Amazonas: near Manaos.

Note: A synonym of $H$. dewnaraini Makhan 1992 fide Makhan (1998: 148); synonymy discussed by Oliva (2000: 14)

decolor [Berosus (Enoplurus)]. Knisch 1924a: 129131: 1 ex. Buenos Aires Prov, in coll. Bruch. Typus and other ex. Paraguay: Santa Trinidad; 1 ex. Bolivia: Yacuiva, coll. Lizer y Trelles and Delétang; $1 \delta^{*}$ Argentina in ZMH, labelled B. occipitalis; name of Régimbart in litt.

1 parat. on a card, with a glass microvial, B. Aires Prov, labelled "Typus"; 1 parat. Paraguay.

Note: There is another $\delta$ on a card, labelled "Cotypus", from Córdoba: Alta Gracia, Knisch det., ex coll. Bruch (see below under decolor in Specimens labelled as types but not included in the original series). Specimens of the Lizer $y$ Trelles coll. not found.

denticulata [Hydrobiomorpha]. Bachmann 1988: 10, 39-40, f. $24 \mathrm{~A}-\mathrm{K}: 1$ O', 1 \% parat. Brasil: Pará State: Alenquer. Holot. $\delta$, allot. $\$, 20 \%, 3 \%$ parat. $i d e m$ in MG; 1 \%, 1 q in coll Bachmann.

$2 \delta, 2 \%$ parat., the $\delta$ with glass microvials, Brasil: Pará: Alenquer, 2 of them ex coll. Bachmann, all pinned.

dilatatus [Tropisternus]. Bruck 1915: 449-450, f. 2: Few ex., Buenos Aires Prov: La Plata. Name of Régimbart in litt.

1 synt., pinned, B. Aires Prov., labelled "Typus" on pale green paper, and "dilatatus Rég. / n. sp. typ." handwritten by Régimbart, ex coll. Bruch.

ethmonotus [Berosus]. Oliva 1989: 78, 117-120, f. 135-141: Holot. $\delta^{\prime}$, allot. $\$, 140$, 16 \% parat. Entre Ríos Prov: Victoria. Other parat. at BM, IML, MZ, USNM.

Holot. of with a glass microvial, allot. $\$, 30$ parat, one of them with a glass microvial, all on cards, E. Ríos: Victoria.

fimbricollis [Spercheus]. Bruch 1915: 460-462, f. 15: 2 ex. Buenos Aires Prov: La Plata; Santa Fe, coll. Richter. 
1 synt. on a card, S. Fe Prov: Chaco, labelled "Typus" on pale green paper, and "Spercheus / fimbriicollis / Bruch" handwritten by Bruch, ex coll. Bruch.

Note: There are 3 synt. from B. Aires Prov at MLP, fide Fernández \& Cabrera (1996: 5), who remark that Bruch recorded only two.

fitthaut [Hemiosus], Oliva 1994a: 278, 290-291, f. $3,16,26,31: 4$ ¿, 5 Q parat. Brasil: Amazonas State: Itú River, near Negro River. Holot. ¿, allot. \$, parat. idem, at MZ [now at INPA]; 2 parat. at BM, 2 parat, at USNM, 2 parat. at ZSM.

17 parat. on cards ( 2 of them on pointed ones), 11 of them with dissected parts glued to the same card, 6 of them with plastic microvials, Brasil: Amazonas: Itu River.

Note: There is a further ex. on a card, labelled "Paratypus" from Brasil: Amazonas State: lower Aripuaná Stream, not mentioned in the original publication, but all labels are authentic.

franca [Hydrobiomorpha]. Bachmann 1988: 8, 36, f. 21 A.H: 1 of parat. Brasil: S. Paulo State: Franca. Holot. idem at MZ.

10 parat., pinned, with a glass microvial, Brasil; S. Paulo: Franca, labelled "Hydrophil./ grandis Régimbart det," handwritten by Régimbart.

gaeae [Tropisternus (Pristoternus)]. Bachmann 1969b: 94-96, ‥ 1-6: Holot . Ó, allot. Q Buenos Aires Prov: Paraná River delta. 1 o, 1 \& parat, idem, in coll. Bachmann.

Holot. of with a glass microvial, allot. 9,2 parat. ex coll. Bachmann, all pinned, B. Aires: Paraná River delta.

Note: Currently in Tropisternus (Tropisternus).

graniformis [Paracymus]. Bruch 1915: 457-458, f. 12: Few ex. Buenos Aires Prov.: La Plata. Name of Régimbart in litt.

1 synt. on a card, B. Aires Prov., labelled "Typus" on pale green paper, and "graniformis Rég./ n. sp. typ." handwritten by Régimbart, ex coll. Bruch.

gravidus [Helochares]. Bruch 1915: 452-453, f. 6: Several ex. Buenos Aires Prov: La Plata; and Formosa: Puerto Bouvier.

3 synt. on cards on 1 pin, B. Aires Prov., labelled "Cotypus" on pale green paper, and "Helochares / gravidus / Bruch" handwritten by Bruch, with a red frame, ex coll. Bruch.

Notes:There are 6 synt. at MLP, fide Fernández \& Cabrera (1996: 5). A synonym of Helochares (Sindolus) femoratus (Brullé 1841), fide Orchymont (1926: 236).

guarani [Enochrus (Methydrus)] Fernández 1990: 87, f. 4, 5: Holot. oै, allot. $\$, 1$ of [and 1 \%?] parat.
Paraguay: Concepción Dept.: Puerto Vallemí $1 \%$ parat. idem at MLP.

Holot. $\delta$, allot. $\$, 2$ parat, on cards, holot. and 1 parat. with glass microvials, Paraguay: Mosquito River, in front of Puerto Vallemí.

guarani [Stethoxus (Stethoxus)]. Bachmann 1966: 12, 14-16, f. 1, 6, 9: Holot. ठ, allot. $ᄋ$ Formosa Prov: Ingeniero Juárez; 4 \&, 4 \% parat. La Rioja Prov.: Patquia, ex coll. Breyer; 1 q Santa Fe Prov: Villa Guillermina; 1 S S. Fe Prov: Calchaquí; 3 ơ S. Fe Prov: Chaco ex coll. Bruch; 28 Tucumán ex coll. Bruch, one of them 15811; 3 o, 2 q Corrientes Prov: S. Cosme. Other parat. from the same localities, and S. Fe Prov: Hersilia, Rosario, and Rafaela; Entre Ríos Prov: Calera Barquin; Córdoba Prov: Leones; Santiago del Estero Prov: Tintina; Brasil: Maranhão: S. Luiz, ex coll. Bachmann. Other parat. at MLP, coll. Daguerre, coll. Jiménez Asúa.

Holot. $\sigma^{2}$, with a glass microvial, allot. $\$$, Formosa: Ing. Juárez; 8 parat. La Rioja: Patquía, ex coll. Breyer; 1 parat. S. Fe: V. Guillermina; 1 parat. S. Fe: Rosario; 1 parat. S. Fe: Calchaqui; 1 parat. S. Fe: Chaco; 1 parat. Córdoba: Leones ex coll. Bachmann; 1 parat. S. del Estero: Tintina ex coll. Jiménez Asúa; 3 parat. (one 15811) Tucumán, ex coll. Bruch and ex coll. Breyer respectiv.; 4 parat. Corrientes: $S$. Cosme; 1 parat. E. Ríos: Calera Barquin; 3 parat. Brasil: Maranhão: S. Luizex coll. Bachmann; all pinned.

Note: Stethoxus Solier 1834 is considered a synonym of Hydrophilus Müller 1764 (Hansen 1991: $170-172,297-298$, f. 60,233 ).

hispidulus [Berosus]. Oliva 1993: 92-93, f. 13-16: 3 ठ, 1 q parat. Brasil: Mato Grosso State: Corumbá. Holot. $\delta$, allot. 9,30 parat. idem, at BM; 1 o at IML, 10 at MZ, 10 at USNM.

$3 \%, 1$ q parat. on cards, one of them with a glass microvial, Brasil: M. Grosso: Corumbá.

iguazu [Beralitra]. Oliva 1996a (1); 38-40, f. 1, 2: Holot. O", allot. 9, 4 parat. Misiones Prov: Iguazú National Park. Other parat. idem, at BM, ISNB, MZ, USNM, ZSM.

Holot. $\delta$, allot., 4 parat. on cards, holot. and 1 parat. with glass microvials, Misiones: Iguazú Nation. Park: San Martín Is.

iguazu [Hydrobiomorpha]. Bachmann 1988:9, 15. 16, f. 31 A-H: Holot. O' Misiones Prov:: Iguazú National Park.

Holot. $\delta$, pinned, with a glass microvial, pinned, Misiones: Iguazú Nation. Park.

inpa [Berosus]. Oliva 1993: 91-92, f. 9-12: 5 ठ, 5 ㅇ parat. Brasil: Amazonas State: Aripuaná River. Holot. o, allot. $\$, 20,2 q$ parat. idem, at INPA. Other parat. at BM, IML, USNM, ZSM. 
$5.8,5$ parat. on cards, the $\delta$ with plastic microvials, Brasil: Amazonas: Aripuaná River.

irinoides [Hydrobiomorpha]. Bachmann 1988: 9 , 30-31, f. 15 A-K: Holot. of Venezuela: Barinas State: Santa Bárbara; allot. $\$, 1 \delta$ parat. Venezuela: Amazonas Fed. Terr.: Dept. Atures, ex coll. Bachmann.

Holot. ơ Venezuela: Barinas: S. Bárbara; allot. Q, 1 o parat. Venezuela: Terr. Fed. Amazonas: Atures Dept., both pinned, all ex coll. Bachmann.

irinus [Hemiosus]. Oliva 1994a: 276, 285-286, f. 12, 22, 30: Holot. ठ", allot. $\%, 4$ O', 6 \% parat. Misiones Prov. Bernardo de Irigoyen: Uruguá-1 River. Other parat. (one in each), idem, at BM, MNHN, MZ [now at INPA], ZSM.

Holot. 0 , allot. 0,11 parat. on cards, 4 of them with dissected parts glued to the same cards, the holot. and 4 o parat. with plastic microvials, Misiones: B. de Irigoyen: Uruguá-1 River.

kalahariensis [Berosus (s. str)]. Orchymont 1936: 115: Holot. O', 30 parat. Kalahari: Nkate, Makarikai; 112 parat. Tsotsoroga Pan; 1 \% from Kabulabula: Chobe River; 1 \& from Makarikari, Nata River, all at TM.

1 parat. on a card, NKate, Makarikari.

laevicollis [Hemiosus]. Oliva 1994b: 75-77, f. 1, 3, 5-9: 5 parat. Venezuela: Bolivar State: Middle Orinoco River: Cuba Island. Holot. $\delta$, 30 parat. idem, at BM.

5 parat. on cards, 4 of them with plastic microvials, Venezuela: Bolívar: Middle Orinoco River: Cuba Is.

limbatus [Paracymus]. Wooldridge 1973: 120-121, f. 5: Holot. $\delta$, allot. $\%, 45$ ठै, 42 \% parat. Venezuela: Guárico State: S. Fernando, at USNM, paratypes Venezuela: Barinas State; Bolivia: S. Cruz Dept.; Brasil: Bahia, Mato Grosso, Pará, Rio Grande do Norte, and S. Paulo States; Colombia: Meta Provi; Paraguay: Asunción, Cordillera Dept., San Bernardino; Perú: Amazonas, Huánuco, and Loreto Depts.; Suriname; Trinidad; Portuguesa, all these at CAS.

1 parat, specimen missing on the card, Venezuela: Guárico: S. Fernando.

longispina [Tropisternus (Pristoternus)]. Fernández \& Bachmann 1981: 272-273, f. 1-6: Holot. of, allot. $\$, 5$ d, 5 q parat. Chaco Prov: San Bernardo; 4 parat. Salta Prov: Acambuco Valley; 1 parat. Salta Prov: 'Tartagal; 1 parat. 27343 Salta Prov: Metán; 4 parat. Formosa Prov.: Ingeniero Juárez; 5 parat. (four 7725, one 27343) Chaco Prov.; 1 parat. Santiago del Estero, ex coll. Bruch; 3 parat. La Rioja Prov:: Patquía, ex coll. Breyer; 1 parat. Córdoba Prov.: Capilla del Monte; 4 parat. Corrientes Prov: S. Cosme. Other parat. from the same and several other localities at CEA, IML, MLP, and coll. Bachmann.

Holot. of with a glass microvial, allot. $\%, 3$ parat. Chaco: S. Bernardo; 8 parat. Salta: Acambuco Valley; 1 parat. Salta: Tartagal; 1 parat. Salta: Rivadavia Dept.: El Palmar, ex coll. Bachmann; 4 parat. 7725, Chaco; 4 parat. Formosa: Ing. Juárez; 1 parat. Santiago del Estero, ex coll. Bruch; 3 parat. S. Estero: Weisburd, ex coll. Bachmann; 1 parat. Córdoba: Capilla del Monte; 1 parat. Córdoba: Morteros, ex coll. Bachmann; 1 parav. La Rioja: Patquía, ex coll. Breyer; 1 parâ. Corrientes; 3 parat. Corrientes: S. Cosme; 5 parat. Entre Ríos: Primero de Mayo, ex coll. Bachmann; 4 parat. E. Ríos: Lazo, ex coll. Bachmann; 1 parat. Buenos Aires Prov., ex coll. Bruch.

Note: Currently in Tropisternus (Tropisternus).

longus [Hydrophilus]. Bruch 1915: 448-449, f. 1: Several ex. Santa Fe: La Gallareta. Name of Régimbart in litt.

Lectot. \%, pinned, S. Fe Prov., labelled "Typus" on pale green paper, and "Hydrophilus / longus / Bruch" handwritten by Bruch, with a red frame; 1 paralectot. $\%$, somewhat teneral, pinned, Chaco [of Santa Fe Prov.], labelled "Typus" on pale green paper, "Hydrophilus / longus / Rég. n. sp. typ.", handwritten by Régimbart, and "Hydrophilus / longus / Bruch" handwritten by Bruch, with a red frame, both ex coll. Bruch.

Notes: Transferred to Neohydrophilus by Orchymont (1919:161-162); to Hydrobiomorpha by Mouchamps (1959: 324, 333); lectot. and paralectot. designated by Bachmann (1988: 1921 , f. 7 A-H).

margaritinus [Berosus]. Oliva 1999: 240-241, 243, f. 1, 3-10; 1 d, 1 \& parat. Brasil: Rio de Janeiro State: Itatiaia National Park. Holot. $\delta^{\circ}$ idem at MZ; $1 \delta^{*}$ parat. at BM.

$16,1 \%$ parat. on cards (one on a pointed one), Brasil: R. de Janeiro: Itatiaia Nation. Park.

meridionale [Pelosoma]. Bruch 1915: 468-469, $\mathrm{f}$ 20: Several ex., Buenos Aires Prov: La Plata, always among rotting plants. Name of Régimbart in litt.

3 synt, on cards on 2 pins, labelled "Typus" and "Cotypus" respectively on pale green paper, B. Aires Prov., the "Typus" labelled "Pelosoma/ meridionale Rég." handwritten by Régimbart.

Notes: There is 1 parat. at MLP, fide Fernández \& Cabrera (1996: 6). A synonym of P. lafertei (Mulsant 1844) fide Orchymont (1941: 14).

mesostitialis [Helochares (Sindolus)]. Fernández de Morras 1981: 189-191, f. 1-3: 4 parat. Buenos Aires Prov, ex coll. Bruch; 1 parat. Córdoba Prov: Alta Gracia, ex coll. Bruch; 2 parat. Formosa Prov: Clorinda; 1 parat. Santiago del Estero Prov.: Airport; 15 parat. Santa Fe Prov: Colonia Macías. Holot. 8 , allot. $\%$, several parat. at MLP; other parat. from the same and several other localities at IML, and coll. Bachmann (B. Aires Prov: Paraná River delta; S. Fe. Prov: Madrejón Don Felipe, and El Toba River; Entre Ríos Prov: Victoria; Corrientes Prov: Carambolas River; Chaco Prov: Ortega Rivulet, 
S. Bernardo, and Resistencia; Formosa Prov.: Negro River, He-He Grande and He-He Chico Streams, Pilcomayo Nation. Park, etc.).

13 parat. on cards, on 8 pins, S. Fe: Garay Dept.: Col. Macias; 1 parat. S. del Estero: Airport; 1 parat. Formosa: Clorinda: Pilcomayo River, ex coll. Bachmann; 37 parat. on cards, on 15 pins, B. Aires; 28 parat. in alcohol in 3 vials, B. Aires: Paraná River delta, ex coll. Bachmann; 4 parat. $\mathrm{S}$. Fe (at light); 1 parat. $\mathrm{S}$. Fe (city); 3 parat., 2 of them with glass microvials, S. Fe: Madrejón Don Felipe, ex coll. Bachmann; 2 parat. Chaco: Ortega Creek, ex coll. Bachmann.

Note: there is a further ex. labelled Paratypus" from Paraguay: Concepción Dept.: Puerto Vallemí, ex coll. Bachmann, not mentioned in the original publication, but the labels are authentic.

min: [Helochares (Sindolus)]. Fernández 1982b: 89 90, f. 7, 9: $q$ parat. Formosa Prov: Clorinda. Holot. $\delta$, allot. Q, Chaco Prov: Barranqueras: La Cava, at MLP. 1 parat. Tucumán at IML, 2 parat. Formosa in coll. Bachmann.

1. $\delta, 1 q$ parat. on cards, the $q$ on a pointed one, the $\delta$ on its side, with a glass microvial, Formosa: Clorinda, ex coll. Bachmann.

misionensis [Enochrus (Methydrus)]. Femández 1990: 87-88, f. 6-8: Holot. ơं, Misiones Prov. Uruguaí River.

Holot. $\mathbf{0}$, dissected parts glued to 2 cards, with a glass microvial, Misiones: Uruguaí River.

missionum [Tropisternus (Tropisternus)]. Fernández \& Bachmann in Femández, Bachmann \& Archangelsky 2001: 193-194, f. 13-15. Holot. $\delta$, 8 parat, Misiones Prov: Bonpland, 3 parat. Misiones Prov: Concepción Dept.: Santa Maria, and Oberá Dept.: Barra Bonita; I parat. Paraguay: Coronel Bogado, ex coll. Bachmann; 1 parat. without precise locality.

Holot. $\delta$ with a glass microvial, 9 parat. (one without head and pronotum), all pinned, Misiones Prov: Bonpland; 1 parat. Misiones Prov: Barra Bonita, ex coll. Bachmann; 2 parat. Misiones Prov: Concepción Dept.: Santa María; 1 parat. Paraguay: Coronel Bogado, ex coll. Bachmann.

monstrosus [Hemiosus]. Oliva 1994a: 278, 292-293, f. 4, 9, 17, 20, 27, 31: 7 d, 8 q parat. from Brasil: Amazonas State: Itu River, near Negro Stream. Holot. $\delta$, allot. $q$ at $\mathrm{MZ}$ [now at INPA]. Other parat. (a couple in each), idem, at BM, USNM, ZSM

13 parat. on cards (one of them on a pointed one, with an elytron glued to a further pointed card), 5 of them with dissected parts glued to the same cards, 7 of them with plastic microvials, Brasil: Amazonas: Itu River, near Negro River. mucajai [Hydrobiomorpha]. Bachmann 1988: 9 , 16-17, f. 4 A-I: 1 o parat. Venezuela: Terr. Fed. Amazonas: High Ventuari River, ex coll. Bachmann. Holot. $\delta, 1$ parat. Brasil: Roraima: Caracaraí, at $\mathrm{MG}$.

1 of parat. Venezuela: Amazonas: high Ventuari River; 1 o parat., Brasil: Caracara River, both pinned and with glass microvials.

multicarinatus [Berosus]. Oliva 1989: 79, 104-105, 198, f. 94-99: Holot. $\delta^{*}$, allot. $, 68,3$ g parat. Salta Prov: Martínez del Tineo. Other parat. idem, at IML, and USNM.

Holot. $\delta$ with a plastic microvial, allot. 9,8 parat, all on cards, Salta: M. del Tineo.

multicostates [Hydrochus]. Oliva 1996b:309, 316319, f. 13, 14: Holot. $\delta$, allot. $Q$ and 79 parat. Buenos Aires Prov: Paraná River delta. Other parat. at BM, ISNB, MZ, USNM, ZSM.

Holot. $\delta$, allot. $\$, 3$ parat. on cards, with dissected parts glued to the same cards (except one parat.), 1 parat. with a plastic microvial, B. Aires Prov.: Paraná River delta.

Note: A synonym of $H$. merkli Makhan 1993 ficle Makhan (1998: 148); synonymy discussed by Oliva (2000: 14).

nitidissimus [Berosus]. Oliva 1989: 79, 169-171, 198, f. 273-280: 4 \%, 2 \% parat. Brasil: M. Grosso State: Corumbá. Holot. $\delta$, allot. $ᄋ, 11 \delta, 1$ \& parat. idem, at BM.

6 parat. on cards ( 3 with plastic microvials), Brasil: M. Grosso: Corumbá.

noa [Tropisternus (Tropisternus)]. Fernández \& Bachmann in Fernández, Bachmann \& Archangelsky 2001: 187-192, f. 1-5, 28-38. Holot. ó, allot. $q$, 10 parat. Tucumán Prov.: Horco Molle, ex coll. Bachmann; parat. Tucumán Prov: Quebrada de los Sosas (3 ex coll. Bachmann), San Pedro de Colalao (2 ex coll. Bachmann), La Criolla (1), Siambón (1), without precise locality (5, 1 of them ex coll. Bruch). Other parat from Salta Prov. at MML; 2 parat. Cordoba Prov. at ISNB, and from several localities in Tucumán, Catamarca, Córtoba, Jujuy and Salta Prov at MLP.

Holot. $\delta$ with a plastic microvial, allot. $q$, Tucumán: Horco Molle, ex coll. Bachmann; 3 parat. Tucumán: Quebrada de los Sosas, ex coll. Bachmann; 3 parat. Tucumán: S. Pedro de Colalao (one 4362, 2 ex coll. Bachmann); 1 parat. Tucumán: Siambón, ex coll. Bruch; 1 parat. Tucumán: La Criolla, labelled "Tropisternus/ nigrinus Shp./v. geniculatus/Klug"handwritten by Bruch, ex coll. Bruch; 5 parat. (one 11847, 4 ex coll. Bruch, one of these labelled "obesus Rég." handwritten by Régimbart), Tucumán; 1 parat. 15818 Tucumán. All pinned. 
obesus [Tropisternus]. Bruch 1915: 450-451, f. 3 : Buenos Aires Prov: La Plata, and Santa Fe Prov: La Gallareta. Name of Régimbart in litt.

1 synt., pinned, B. Aires Prov., labelled"Typus" on pale green paper, and "Tropisternus / obesus Rég." handwritten by Régimbart, ex coll. Bruch.

obsoletus [Philhydrus]. Bruch 1915: 453-454, f. 7: Several ex. Buenos Aires Prov: La Plata. Name of Régimbart in litt.

Lectot. on a card, labelled "Typus" on pale green paper, and "obsoletus Rég. / n. sp. typ." handwritten by Régimbart; 6 paralectot. on cards (on 3 pins), labelled "Cotypus" on pale green paper, B. Aires Prov, ex coll. Bruch.

Notes: There is 1 paralectot. at MLP, fide Fernández \& Cabrera (1996: 7). Transferred to Enochrus (Lumetus) by Knisch (1921a: 70), and to $E$. (Methydrus), and lectot. and paralectot. designated by Fernández (1989b: 124-125, f. 8-11).

orchymonti [Hydrochus]. Oliva 1996b: 310, 313316: Holot. $\delta$, allot. 0,6 o parat. Buenos Aires Prov: Paraná River delta. Other parat. at BM, USNM, ZSM.

Holot. $\delta$, allot. $\$, 6 \delta^{t}$ parat. on cards, dissected parts glued to the same cards (except one parat.), all with plastic microvials, B. Aires Prov: Paraná River delta.

Note: A synonym of $H$ coeneni Makhan 1992 , fide Makhan (1998: 148); synonymy discussed by Oliva (2000: 14).

paraensis [Enochrus [(Methydrus)]]. Fernández 1997: 25, f. 15 17, 26: Holot. ठ", allot. o, 2 parat., Brasil: Pará State: Belém: Manatíes basin.

Holot. 8 , allot. \&, 2 parat., dry preserved, in glass microvials, Brasil: Pará: Belém.

paranensis [Derallus]. Oliva 1982: 292-294, pl. i: f. $6 \mathrm{a}, \mathrm{b}$, ii: f. $6 \mathrm{a}, \mathrm{b}$ : Holot. 0 , 10 parat. Buenos Aires: Paraná River delta. Other parat. at IML, MLP, USNM, coll. Bachmann, coll. Oliva.

Holot. $\delta, 19$ parat. on cards, holot. and 1 parat. with glass microvials, B. Aires: Paraná River delta, 5 of them ex coll. Bachmann.

paulista [Hydrobiomorpha]. Bachmann 1988: 9, 33-34, f. 18 A-I: 1 \% parat. Brasil: S. Paulo State: Ribeirão Preto. Holot. $\delta^{*}$, allot. $\$$ Brasil: S. Paulo State: Franca, at MZ. Preto.

1 \% parat., pinned, Brasil: S. Paulo: Ribeirão

pauxillus [Berosus (s. str.)]. Knisch 1924a: 136-137: $14 \delta$ and $q$ Buenos Aires Prov,; 1 ex. Tucumán Prov. in coll. Bruch.

3 synt. on cards on 1 pin, with a glass microvial, B. Aires Prov, labelled "Cotypus" on pale green paper, and "Knisch det. / pauxillus m." handwritten by Knisch, ex coll. Bruch.
Note: A synonym of Berosus minimus Knisch 1921b, B. pauxosus Orchymont 1943, and B. stratonicus Orchymont 1946, fide Oliva (1989: 78, 107-110, f. 108-115).

pectoralis [Derallus]. Oliva 1983b: 343-344, pl. i: f. 1 a-c, ii: f. $1: 3$ \&, 1 ơ parat. Brasil: Pará State: Belém. Holot. $\delta$, allot. idem at INPA; $1 \delta^{\circ}$ at BM, $1 \delta, 1 \%$ at USNM; 10 at ZSM; 10 , 1 \% temporarily in coll. Oliva, now deposited at MACN.

6 parat. on cards, 2 of them with glass microvials, Brasil: Pará: Belém: Manatíes Basin, 2 of them ex coll. Oliva, deposited at MACN.

perpunctata [Helobata]. Fernández \& Bachmann 1987: 156-158, f. 15, 16: Holot. 6 , allot. $\$, 1$ б, 4 \% parat. Chaco Prov:: S. Bernardo, at light.

Holot. 8 , allot. $\%, 10,3 \%$ parat., holot. and 1 parat. with glass microvials, all in alcohol in 3 vials, Chaco: S. Bernardo.

perpunctatus [Derallus]. Oliva 1983b: 346-348, pl. i: f. 3a-f, ii: f. $3: 1 \delta^{\prime \prime}, 3$ \% Brasil: Amazonas State: Igarapé Cachoeira near Cueiras River. Holot. $\delta$, allot. $\$$ idem, at INPA; 19 at BM; 1 $\delta, 1$ q parat. at USNM; 1 q at ZSM; 1 in coll. Oliva.

$1 \delta, 3$ parat. on cards, the $\delta$ with a glass microvial, Brasil: Amazonas: Cueiras River: Igarapé Cachoeira, at light.

phallicus [Berosus] Oliva 1989: 81, 181-182, f. 312320: Holot. $\delta$, allot. $\$, 2$ ठ, 1 \% parat. Buenos Aires Prov.: Ingeniero Otamendi. Other parat. idem at BM.

Holot. $\delta^{*}$, allot. $\%, 2 \delta^{*}, 1 \%$ parat., holot. and $\delta^{\circ}$ parat. with plastic microvials, all on cards, $B$. Aires: Otamendi.

pichilingue [Helochares (Helochares)]. Fernández 1989a: 147, f. 4-7: Holot. ${ }^{\circ}$ Ecuador: Los Rios Prov: Quevedo: Pichilingue River.

Holot. tin a glass vial, with a glass microvial, Ecuador: Los Ríos: Quevedo, Pichilingue River.

pseudosecretus [Hydrochus]. Oliva 1996b: 306, 321-322, f. 18-20: Holot. $\delta^{*}$, allot. $\$, 4$ ठै, 2 \% parat. Venezuela: Barinas State: Santa Bárbara. Other parat. at BM, ISNB, MZ, USNM, ZSM.

Holot. $\delta$, allot. $\$, 6$ parat. on cards, dissected parts glued to thew same cards, holot. and 3 parat. with plastic microvials, Venezuela: Barinas: S. Bárbara.

Note: A synonym of $H$. purpureus (Knisch 1921) fide Makhan (1998: 148); synonymy discussed by Oliva (2000: 14).

puncticolle [Phaenonotum]. Bruch 1915:467-468: Several ex. Buenos Aires Prov: La Plata. Name of Régimbart in litt.

Lectot., 6 paralectot. on cards, on 3 pins $(1+$ $3+3)$, B. Aires Prov., labelled "Typus", "Cotypus", 
and "Cotypus" respectively on pale green paper, the lectot., in addition, "puncticolle / Rég. n. sp. typ." handwritten by Régimbart, ex coll. Bruch.

Notes: There are 2 paralectot. at MLP, fide Fernández \& Cabrera (1996: 7). Lectot. and paralectot. designated, and recorded as Phaenonotum (Hydroglobus) puncticolle by Archangelsky (1992: 47-50). This is the type species of Hydroglobus Knisch (1921a: 78).

punctipennis [Hemiosus]. Oliva 1994a: 278, 293294, f. 5, 18, 28, 31: Holot. o, allot. 0,1 dै, 3 Q p parat. Uruguay: Artigas Dept.: San Gregorio.

Holot. $\delta$, allot. $\$, 10 \% 3 \%$ parat. on cards, the holot. and 3 parat. with dissected parts glued to the same cards, the holot. and 1 parat. with plastic microvials, Uruguay: Artigas: S. Gregorio, ex coll. Bachmann.

regimbarti [sub Regimbarti] [Phaenonotum]. Bruch 1915: 466-467: Several ex. Buenos Aires Prov: La Plata; = P. Bruchi Régimbart in litt.)

Lectot. $\delta^{*}, 2$ paralectot. on cards on 2 pins, lectot. and 1 paralectot. with plastic microvials, B. Aires Prov., labelled "Typus" and "Cotypus" respectively on pale green paper, the lectot., in addition, "Bruchi Rég. / n. sp. type" handwritten by Régimbart, ex coll. Bruch.

Notes: There are 3 paralectot. at MLP, fide Fernández \& Cabrera (1996:7). Lectot. and paralectot. designated by Archangelsky (1991: 158-159, f. 1-3, 7).

richteri [sub Richteri] [Hydrochus]. Bruch 1915: 463-464, f. 17: Few ex. Buenos Aires Prov: La Plata; 10 ex. Santa Fe, coll. Richter.

Lectot., 1 paralectot. on cards, B. Aires Prov., labelled "Typus" and "Cotypus" respectively on pale green paper, and "Hydrochus / Richteri / Bruch" handwritten by Bruch, ex coll. Bruch.

Notes: There are 4 paralectot. at MLP, fide Fernández \& Cabrera (1996: 7-8). Lectot. and paralectot. designated by Oliva (1992: 90, 99-100, f. 8,30 ), who says $H$. soesilaae Mackan 1994 is a synonym. Included in Rishwanius by Makhan (1998: 146, f. 17). Rishwanius is considered a synonym of Hydrochus by Oliva (2000: 14).

rufocinctus [Paracymus]. Bruch 1915: 456-457, f. 11: Many ex. Buenos Aires Prov: La Plata. Name of Régimbart in litt.

4 synt., 13 parat. on cards, labelled "Typus" and "Cotypus" respectively on pale green paper, B. Aires Prov; one of the synt. in addition "Paracymus / rufocinctus / Rég. n. sp. typ." handwritten by Régimbart; all ex coll. Bruch.

Note: There are 4 synt. at MLP, fide Fernández \& Cabrera (1996: 8). rufulus [Berosus (s. str)]. Knisch 1924a: 135-136: 1 9 Mendoza, 1 t Santiago del Estero, both in coll. Bruch; 1 \& Tucumán, leg. Bruch, in coll. Knisch.

10 synt. with a glass microvial, Santiago del Estero Prov., labelled "Paratypus" [error?], 1 \% synt. Mendoza Prov., labelled "Cotypus" on pale green paper, and "Berosus / Kniž det. rufulus Kn." handwritten by Knisch, both on cards, ex coll. Bruch.

Note:There is another specimen from Córdoba: Alta Gracia, ex coll. Bruch, labelled "Cotypus" on pale green paper, and "Knisch det. 1922/ Berosus s. str. / rufulus Knisch" handwritten by Knisch, not mentioned in the original publication (see below under rufulus in Specimens labelled as types but not included in the original series).

sanapana [Tropisternus (Pristoternus)]. Bachmann 1970: 59-61, f. 5-8: Holot. $\delta$, allot. $Q$, 10 parat. Paraguay: Concepción Dept.: Puerto Vallemí. Other parat. idem, at MLP, and coll. Bachmann.

Holot. 8 , allot. 9,25 parat, pinned, holot. and of parat. with glass microvials, Paraguay: Concepeión: P. Vallemí, all ex coll. Bachmann.

Note: Pristoternus was synonymized with Tropisternus (Tropisternus) by Hansen (1989: 54); the name was revalidated as a subgenus of Tropisternus by Bachmann (1993: 50, 72).

sapucay [Chasmogenus]. Fernández 1988: 192-193, f. 3-9: Holot. $\delta$, allot. $\$, 6$ parat. Paraguay: Sapucay. Other parat. from the same locality and Argentina: Formosa Prov: Clorinda, and Santiago del Estero, at BM, IML, MLP.

Holot. $\delta$ with a glass microvial, allot. $\$, 6$ parat. ( 5 on cards on 1 pin; 1 in a glass vial), Paraguay: Sapucay.

sapucay [Tropisternus mutatus, subsp.]. Fernández \& Bachmann, in Fernández, Bachmann \&Archangelsky 2001: 195-196, f. 22-27. 5 parat. Salta Prov: Pocitos, $e x$ coll: Bachmann; 10 parat. Salta Prov: Aguas Blancas, ex coll. Bachmann; 10 parat. Jujuy Prov: Yuto, ex coll. Bachmann; 10, 2 \% parat. Jujuy Prov: between Ledesma and Valle Grande, ex coll. Bachmann; 1 parat. Tucumán Prov: Choromoro, ex coll. Bachmann; 20 parat. Misiones Prov: Bonpland, labelled "Typus" and "Cotypus" (of $T$. mutatus Orchymont) ex coll. Bruch; 3 parat. Misiones Prov., without precise locality, labelled "Typus" (1) and "Cotypus" (2) (of T. mutatus) ex coll. Bruch; 10 parat. Misiones Prov: Puerto Berbus; 4 parat. Corrientes Prov: Santo Tomé, ex coll. Pellerano; 2 ơ parat. Bolivia: NorYungas Prov: Capari; $1 \%$ parat. Paraguay, no precise locality, labelled "Cotypus", ex coll. Bruch. Holot. $\delta^{*}$, allot. \&, 2 \& parat. Paraguay: Sapucay, labelled "Paratype" (of T. mutatus); 1 \% parat. Misiones Prov: Bonpland labelled "Cotype" (of T. mutatus); 1 \% parat. Misiones Prov: near San Ignacio labelled "Cotype" (of $T$. mutatus); 1 to parat. Paraguay, no precise locality, labelled "Cotype" (of 7". mutatus) ex coll. Bruch, all these labelled by Orchymont, at ISNB. Thirteen other parat. from Jujuy and Misiones Prov at MLP. 
5 parat., pinned, Misiones, one of them labelled "Typus", one "Cotypus" [of T, mutatus] on pale green paper, ex coll. Bruch; 2 Misiones: Bonpland, 1 labelled "Cotypus" [of T. mutatus] on pale green paper, ex coll. Bruch; 1 parat. 36899 with a glass microvial, Misiones: Puerto Bemberg [now called Puerto Libertad]; 4 parat. Corrientes: Santo Tomé, ex coll. Pellerano; 1 parat. Tucumán: Choromoro, ex coll. Bachmann; 3 parat. Jujuy: between Ledesma and Valle Grande, ex coll. Bachmann; 1 . parat. Jujuy:Yuto, ex coll. Bachmann; 1 parat. with a glass microvial, Salta: Aguas Blancas, ex coll. Bachmann; 4 parat., one with a glass microvial, Salta: Pocitos; 2 parat. Bolivia: Nor Yungas: Caraparí; 1 parat. Paraguay, ex coll. Bruch.

sculptile [Pelosoma]. Orchymont 1937:249-250: Brasil: S. Paulo, holot. 0 at MP; a parat. series; 1 ex. at ZMB.

1 parat. 40375 on a card, Brasil: S. Paulo, labelled "Para / type" on pink paper, and "A. d'Orchymont det. / Pelosoma / sculptile m." handwritten by Orchymont; ex coll. Bruch.

sculptipennis [Hemiosus]. Oliva 1994a: 282, 294-

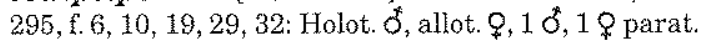
Entre Ríos Prov.: Colón Dept.: Mendoza creek; 2 \& parat. E. Ríos Prov: Concordia Dept.: Yuqueri Grande creek; 3 o', 6 q parat. Entre Ríos Prov: Victoria Dept., Manantiales Creek. Other parat. (a couple in each) at BM, MZ [now at INPA], USNM, ZSM.

Holot. ${ }^{\circ}$, allot., $1 \delta^{*}$ parat. on cards, the holot. and the parat. with dissected parts glued to the same cards, the holotype, in addition, with a plastic microvial, E. Ríos: Colón: Mendoza Creek; 1 parat. on a card, E. Ríos: Concordia: Yuquerí Grande Creek; 4 parat. on cards, one of them with a plastic microvial, E. Ríos: Victoria: Manantiales Creek; 10 parat. on cards, 4 of them with plastic microvials, E. Ríos: Victoria.

sculptum [Oosternum]. Bruch 1915: 469-470, f. 21 : Few ex. Buenos Aires, among rotting tomatoes in an orchard. Name of Régimbart in litt.

5 synt. on cards, on 4 pins, B. Aires Prov., 2 labelled "Typus", 2 "Cotypus" on pale green paper, one of the Typi labelled "sculptum Rég. / n. sp." handwritten by Régimbart, cut, with only remainings of "type".

Notes: There are 9 synt, at MLP, fide Fernández \& Cabrera (1996: 8). Transferred to Pemelus by Malcolm (1981, unpubl. thesis), and by Hansen (1991: 266).

scutellaris [Philhydrus]. Bruch 1915: 455, f. 4: Buenos Aires Prov: La Plata, a fairly common species.

Lectot. $\delta, 8$ paralectot., on cards, on 6 pins, $B$. Aires Prov., two of the paralectot. labelied "Typus", lectot. and the remaining paralectot. labelled
"Cotypus" on pale green paper, all labelled "Philhydrus / scutellaris / Bruch" handwritten by Bruch, ex coll. Bruch.

Notes: There are 4 paralectot, at MLP, fide Fernández \& Cabrera (1996: 8). Transferred to Enochrus (Lumetus) by Knisch (1924b: 214); to Enochrus (Hugoscottia) by Orchymont (1937: 354). Lectot. and paralectot. designated by Fernández (1989b: 122-123, f. 1-4).

seriatus [Berosus]. Berg 1885: 222-223: 3 ex. Buenos Aires ( 1 at the UBA, 2 in coll. Berg).

1 synt. on a card, with a glass microvial, B. Aires, labelled "Typus" (printed with red ink on white paper); on the bottom of the drawer a label reads: "seriatus / Berg / R. A." [R. A. means República Argentina] handwritten by Berg.

Notes:There is 1 synt. at MLP, fide Fernández \& Cabrera (1996: 8). A synonym of Berosus alternans Brullé 1841 fide Oliva (1983a: 45-46, pl. i: f. $2 \mathrm{a}-\mathrm{h}$; ii: f. $2 \mathrm{a}-\mathrm{c}$ ); the synonymy was already suspected by Knisch (1924b: 268).

sesquirina [Hydrobiomorpha irina, subsp.]. Bachmann 1988: 9, 28-29, f. 13 A-H: 1 ठ, 1 O parat. Brasil. S. Paulo. Holot. $\delta$, allot. $\$, 2$ parat. at MZ.

1 of, 1 o parat., pinned, the of with a glass microvial, Brasil: S. Paulo (city), both with old labels which read "Hydrophil. / politus".

simmonsorum [Guyanobius]. Spangler 1990: 411415, f. 1-11: Parat. Brasil: Pará: small tributary of Xingu River. Holot. $\delta$, allot., parat. idem at MZ, parat. at several institutions.

10', 19 parat. on pointed cards, Brasil: Pará: Xingu River.

sinigus [Berosus]. Oliva 1989: 82, 152-154, f. 233239: Holot. O", allot. $\%, 3$ \%, 9 \% parat. Buenos Aires Prov: Paraná River delta. Other parat. idem at IML, and USNM.

Holot. of with a glass microvial, allot. $\$, 12$ parat., 3 of them with plastic microvials, all on cards, B. Aires: Paraná River delta.

spatula [Hydrobiomorpha]. Bachmann 1988:8,4344, f. 28 A-I: Holot. Ö Brasil: Bahía State: km 965, Encrucilhada, ex coll. Bachmann.

Holot. $\delta$, pinned, with a glass microvial, Brasil: Bahía: $\mathrm{km}$ 965, Encrucilhada, ex coll. Bachmann.

spatulatus [Helochares (Sindolus)]. Fernández de Morras 1981: 191-192, f. 4-7: 1 d parat. Corrientes Prov: Carambolas Creek, ex coll. Bachmann. Holot. of Corrientes Prov: Nueve de Julio, 10 parat. Santa Fe Prov: Colonia Macías, at MLP; 10,2 o parat. Paraguay: Concepción Dept.: Puerto Vallemí in coll. Bachmann. 
5 parat. on cards, on 3 pins $(1+2+2), 1$ of them with a glass microvial, Paraguay: Concepción: P. Vallemí; 3 of them ex coll. Bachmann.

spegazzinii [sub Spegazzinil] [Derallus]. Bruch 1915: 460, 483: Buenos Aires Prov: Los Talas (near La Plata), and Santa Fe; in part at light.

7 synt. on cards, on 5 pins, one of the "Cotypi" with glass microvials; 2 labelled "Typus", 5 "Cotypus" on pale green paper, and "Derallus / spegazzinii / Bruch" handwritten by Bruch, B. Aires Prov, ex coll. Bruch.

Notes: There is 1 synt. at MLP, fide Fernández \& Cabrera (1996: 8). A synonym of Derallus angustus Sharp 1885, fide Oliva (1982: 287-288, pl.i: f. 1a,b, ii: f. 1a,b); the synonymy was already suspected by Knisch (1924b: 278).

studiosonum [Hydrochus]. Oliva 1996b: 305, 312313, f. 1, 2: Holot. o, 1 parat. Buenos Aires Prov, Paraná River delta; allot. $Q$ Buenos Aires Prov., ex coll. Bruch; 1 parat. Entre Ríos Prov: Victoria Dept., Manantiales Stream. Other parat. at BM, USNM, ZSM.

Holot. 8, with a plastic microvial, B. Aires: Paraná River delta; allot. \&, B. Aires Prov., labelled "Knisch det. 1924/Hydrochus/n.sp. prope / squamifer Lec. / Unicum" handwritten by Knisch; 1 parat., dissected parts glued to the same card, with a plastic microvial, as holot.; 1 parat. dissected, all parts glued to a card, with a plastic microvial, all on cards, Entre Rios Prov: Victoria: Manantiales Stream.

Note: A synonym of $H$. granulatus Blatchley 1910 fide Makhan (1998: 148); revalidated by Oliva (2000: 14).

subandinus [Berosus]. Oliva 1989:78, 106-107,198, f. 100-106: Holot. ¿ Salta Prov: Martínez del Tineo; allot. \& La Rioja Prov: Patquía. 20 , 2 \% parat. La Rioja Prov: Patquia, at BM.

Holot. $\delta$ with a plastic microvial, Salta: M. del Tineo; allot. I La Rioja: Patquía, both on cards.

subglobosus [Derallus], Oliva 1983b: $349-350, \mathrm{pl}$. i: f. 5a,c; ii: f. $5: 20^{\circ}$, 1 \% parat. Brasil: Amazonas State, affluent of Moravia River; Sierra Neblina (at Negro Stream). Holot. $\delta^{t}$, allot. $\%$ at INPA. Other parat.: 10 at BM; $10^{\circ}, 1 \%$ at USNM; 10 at ZSM.

3 parat, on cards, 2 of them with glass microvials, Brasil: Amazonas: Rio Negro, $\mathrm{S}^{\mathrm{a}}$. Neblina, tributary of Moravia River.

talarum [Helochares (Sindolus)]. Fernández 1983: 440-444, f. 1-16: 1 \%, 1 \% parat. Buenos Aires Prov: Berisso: Los Talas; 2 parat. 27138, B. Aires Prov: Rosas; 1 parat. B. Aires Prov: San Clemente del Tuyú; 1 parat. Santa Fe. Holot. $\delta$, allot. $q$ B. Aires Prov. Los Talas at MLP; other parat. from the same and other localities in B. Aires and Santa Fe Prov. at IML, MLP, and coll. Bachmann.
3 parat. on cards, 2 of them with glass microvials, B. Aires: Los Talas; 5 parat. on 2 pins $(2+3)$, two 27138 , B. Aires: Rosas; 1 parat. B. Aires: S. Clemente del Tuyú; 10 parat. B. Aires: Chascomús Pond, ex coll. Bachmann; 1 parat. $\mathrm{S}$. Fe City, ex coll. Bachmann.

tartagalensis [Berosus]. Oliva 1995a: 58-60, f. 1 6: Holot. $ठ$, allot. $9,10 \%, 6$ \% parat. Salta Prov: Tartagal. Other parat. idem at BM, MZ, USNM, ZSM.

Holot. 8 , allot. 9,7 parat. on cards ( 7 of them on pointed ones), the holot. and 1 parat. with dissected parts glued to the same cards, and with a plastic microvial, Salta: Tartagal.

tectiformis [Helochares (Helochares)]. Fernández 1982b: 88, f. 5: Holot. $\delta^{\prime \prime}$, allot. $\%, 1 \delta^{\star \prime}$ parat. Corrientes Prov: Santo Tomé, ex coll. Pellerano. Other parat. from Paraguay at MML; from Misiones Prov., and Brasil: Corumbá, at MLP; from Venezuela in coll. Bachmann.

Holot. $\delta^{\circ}$, allot. , 10 parat., holot. and parat. with glass microvials, all on cards, Corrientes: S. Tomé ex coll. Pellerano; 1 parat. Venezuela: Ar [agua?]: Pie del Cerro, ex coll. Bachmann.

terranovae [Derallus], Oliva 1983b: 348-349, pl, i: f. 4a-d; ii: f. 4: 1 б, 1 \% parat. Brasil: Amazonas State: Paraná da Terra Nova (lower Solimões River). Holot. $\delta$, allot. $\&$ at INPA. Other parat.: $1 \%$ at BM; $1 \%$ at ZSM.

$16,1 \%$ parat. on cards, the $\delta$ with a glass microvial, Brasil: Amazonas: lower Solimões: Paraná da Terra Nova.

toxacanthus [Berosus]. Oliva 1989: 82, 147-149, 198, f. 220-225: Holot. o', allot. 9,7 parat. Salta Prov: Los Arrasayales (close to Baritú National Park).

Holot. $\delta^{*}$, allot. 9,7 parat. on cards, holot. and 1 parat, with glass microvials, Salta: close to Baritú Nation. Park.

unguidentatus [Berosus]. Oliva 1989: 80, 178-180, 198, f. 306-311: Folot. Oै, allot. 9 Santa Fe Prov:: Rosario, ex coll. Stevenin; 7 parat. Santa Fe Prov:: Garay: Colonia Macias. Other parat. at IML and BM.

Holot. $\delta$ with a plastic microvial, allot. $\%$, Santa Fe: Rosario, ex coll. Stevenin; 7 parat., 1 of them with a glass microvial, S. Fe: Garay: Col. Macías, all on cards.

ussingi [sub Ussingi] [Berosus]. Jensen Haarup 1910: 543: "Very common in the districts round Mendoza".

1 paralectotype? on a card, labelled "Cotypus" on pale green paper, and "Berosus / ussingi / J.Hrp." handwritten by Bruch, with a red frame, Mendoza Prov., ex coll. Bruch.

Note: The specimen was compared with the types by Oliva (1983a: $49-50$, f. 4 ); she also designated a lectotype from Mendoza: Santa 
Rosa, and said the specimen in Brich's collection may be from the Jensen Haarup series.

utiariti [Hydrobiomorpha]. Bachmann 1988: 8 , 18-19, f. 6 A-K: Holot. O', allot. + Brasil: Mato Grosso State: Utiariti, Papagaio River. 1 \& parat. Paraguay: Concepción Dept.: Puerto Vallemí, in coll. Bachmann.

Holot. of with a glass microvial, allot. $\%$, both pinned, Brasil: M. Grosso: Utiariti, Papagaio River, ex coll. Bachmann; 1 \& parat., Paraguay: Concepción: P. Vallemí, all pinned, ex coll. Bachmann.

utinga [Hydrobiomorpha]. Bachmann 1988: 9, 1.7. 18 , f. 5 A-K: 1 ơ, 1 \& parat. Brasil: Pará State: Belém: Utinga and other localities. Holot. $\delta$, allot. $Q, 1 \delta$ parat. idem, at $M G, 10$ idem, ex coll. Bachmann.

4 parat., pinned, 3 of them with glass microvials, Brasil: Utinga; Brasil: Belém; Brasil: Pará: Tucurul, and Brasil: Pará: Coqueiro, respectively.

varginha [Hydrobiomorpha irina, subsp.]. Bachmann 1988: 9, 29-30, f. 14 A-I: Holot. Oै Brasil: Minas Gerais State: Varginha, ex coll. Bachmann. Allot. O Brasil: Mato Grosso State: high Xingu River: P. Leonardo Agosto, at MZ.

Holot. $\delta$, pinned, with a glass microvial, Brasil: M. Gerais: Varginha, ex coll. Bachmann.

venezolana [Hydrobiomorpha]. Bachmann 1969a: 290-291, f. 14: 16, 1 \% parat. Venezuela: Delta Amacuro Terr: Tobejuba. Folot. $\delta$, allot. $९, 3 \delta, 1$ \% parat. idem, at MLS.

10,1 \% parat. pinned, with glass microvials, Venezuela: Delta Amacuro: Tobejuba.

venezuelensis [Enochrus (Methydrus)]. Fernández 1990: 89, f. 15, 16: Holot. $\delta^{\star}$, allot. 9,10 parat. Venezuela: Caracas.

Holot. $\delta$ with a glass microvial, allot., 1 , parat., all on cards, Venezuela: Caracas.

ventricosus [Helochares]. Bruch 1915: 452, f. 5: Several ex. Buenos Aires Prov: La Plata; one Tucumán. Name of Régimbart in litt.

Lectot., 2 paralectot. on cards, 2 of them, on 1 pin, with a glass microvial each, these labelled "ventricosus Rég. / n.sp. type" handwritten by Régimbart, all labelled "Typus" on pale green paper, B. Aires Prov., ex coll. Bruch.

Notes: There are 2 paralectot. at MLP, fide Fernández \& Cabrera (1996: 9). Recorded under Helochares (Sindolus), and lectot. $\delta$, and paralectot. $\delta$ and $\%$ designated by Fernández (1982a: 36-38, f. 4, 5).

virescens [Eumetacymus]. Brèthes 1922: 264: Buenos Aires.
Holot. on a card, B. Aires, labelled "Eumetan cymus / virescens / Brèthes" and "type?", handwritten by Brèthes, with a small square piece of red paper.

Note: A synonym of Paracymus rufocinctus Bruch 1915, fide Orchymont (1938: 265).

\section{NAMES NOT FOUND IN THE LITERATURE AND PROBABLY NOT PUBLISHED}

\section{bruchi [Berosus]. Régimbart}

1 ex. on a card, labelled "Typus" on pale green paper, "Berosus / Bruchi Rég." handwritten by Régimbart, and "Knisch det. 1922 / Berosus s. str. / bonaerensis Bg.", handwritten by Knisch, Buenos Aires Prov., ex coll. Bruch.

Note: It is Berosus chalcocephalus Germain 1856 , which has $B$. bonaerensis Berg 1885 as a synonym.

\section{dehiscens [Berosus]. Régimbart}

2 ex. on cards, on 1 pin, with a glass microvial, Buenos Aires Prov, labelled "Typus" on pale green paper and "dehiscens Rég. / n. sp. type" handwritten by Régimbart, ex coll. Bruch.

Notes: The name was later published by Jensen Haarup (1910: 542, 544), based on 6 syntypes from Mendoza Prov., housed in Mus. Köbenhavn; 1 of these was designated lectotype by Oliva (1983: 51-52, pl. i: f. 6 a-c, ii: 6 a-c); 3 were identified by her as Berosus pallipes. B. Aires was not mentioned in the original publication. The present specimens are Berosus pallipes Brullé 1841.

richteri [Berosus]. Régimbart.

1 ex. on a card, Buenos Aires Prov, labelled "Richteri Rég. / n. sp. type" handwritten by Régimbart, and "Knisch det. / bonaerensis Bg" handwritten by Knisch, ex coll. Bruch.

Note: The ex. is B. chalcocephalus Germain 1856, which has B. bonaerensis Berg 1885 as a synonym.

sellatus [Philhydrus]. Régimbart.

1 ex. on a card, Buenos Aires Prov., labelled "Typus" on pale green paper, and "Philhydrus / sellatus Rég." handwritten by Régimbart.

\section{SPECIMENS LABELLED AS TYYPES BUT NOT INCLUDED IN THE ORIGINAL SERIES}

adustus [Berosus]. Knisch 1922a: 116-117: 6 ex. Buenos Aires, 1920 at ZMH; 1 \% Uruguay: Montevideo, at ZMB

1 o B. Aires Prov., 1905, labelled "Cotypus" on pale green paper, and "Knisch det. 1923/ 
Berosus O / adustus / Kn." handwritten by Knisch, ex coll. Bruch.

Note: The specimen was not mentioned in the original publication.

argentinus [Hydrobius (Hydramara)], Knisch 1925: 2-4: 3 ex. Mendoza Prov: Punta del Agua, Jensen Haarup; 4 ex. [Salta Prov.?]: Tala, leg. Borelli.

3 ex., pinned, Catamarca Prov.: Corral Quemado; Jujuy, and Catamarca Prov: Hualfin, labelled "Typus", "Typus" and "Cotypus" respectively on pale green paper, one of them labelled "Knisch det. 1925 / Hydrobius / argentinus Kn." handwritten by Knisch, ex coll. Bruch.

Notes:These specimens and localities were not mentioned in the original publication. Recorded as Hydramara argentina by Orchymont (1942:25).

atratus [Helochares]. Bruch 1915: 451-452, 481, f. 4: Several ex., Buenos Aires Prov: La Plata, and B. Aires. Name of Régimbart in litt.

1 ex. on a card, Misiones Prov., labelled "Cotypus", ex coll. Bruch.

Note: Misiones and this specimen were not mentioned in the original description (see above under atratus in Catalog of the types).

bruchi [Hemiosus]. Knisch 1924a: 139-140: 8 ex. (3 in coll. Knisch), Córdoba Prov: Alta Gracia, coll. Bruch.

2 ex. on cards, Córdoba: Alta Gracia, labelled "Cotypus" on pale green paper, one of them, in addition, "Knisch det. 1924 / Hemiosus / Bruchi Knisch" handwritten by Knisch, ex coll. Bruch.

Note: The 5 synt. mentioned in the original publication are all labelled "Typus"; these ones were not mentioned. See above under bruchi in Catalog of the types.

cornicinus [Berosus]. Knisch 1922a: 118-119: 2 우 "Brasil", in ZMB.

1 ex. on a card, Córdoba Prov.: Alta Gracia, labelled "Cotypus" on pale green paper, and "Knisch det. 1923 / Berosus cor- / nicinus m. (var.?)" handwritten by Knisch.

Notes: Córdoba was not mentioned in the original publication; moreover the specimen was labelled by Knisch after the publication date.

decolor [Berosus (Enoplurus)]. Knisch 1924a: 129131: 1 ex. Buenos Aires Prov, in coll. Bruch. Typus and other ex. Paraguay: Santa Trinidad; 1 ex. Bolivia: Yacuiva, coll. Lizer y Trelles and Delétang; $1 \delta$ Argentina in ZMH, labelled B. occipitalis; name of Régimbart in litt.

20 on cards, one Paraguay, the other Córdoba: Alta Gracia, both labelled "Kniž det. / decolor m." handwritten by Knisch, ex coll. Bruch.
Note: These specimens were not mentioned in the original publication, see above under decolor in Catalog of the types.

rufulus [Berosus]. Knisch 1924a: 135-136: 1 q Mendoza, 18 from Santiago del Estero, both in coll. Bruch; 1 \& from Tucumán, leg. Bruch, in coll. Knisch.

1 ex. on a card, Córdoba Prov.: Alta Gracia, labelled "Cotypus" on pale green paper, and "Berosus / Kniž det. rufulus Kn" handwritten by Knisch, ex coll. Bruch.

Note: The specimen and the locality were not mentioned in the original publication; see above under rufulus in Catalog of the types.

speciosus [Berosus (Enoplurus)]. Knisch 1921b: 67: A short series ( $\delta$, $q$ ) Brasil: Mato Grosso State: Corumbá, in coll. Knisch.

1 \% on a card, with a glass microvial, Argentina: Santa Fe Prov, labelled "Cotypus" on pale green paper, and "Berosus 60 / (Enoplurus) / speciosus $\mathrm{m}$. / Kniž det." handwritten by Knisch, ex coll. Bruch.

Note: Santa Fe was not mentioned in the original publication.

tremolerasi [Hugoscottia]. Knisch 1922b: 104, 105: Uruguay: Menafra.

4 ex. on cards, on 2 pins $(1+3)$, Córdoba, and Córdoba Prov.: Alta Gracia, both labelled "Cotypus" on pale green paper, one of them, in addition "Knisch det. 1923/ Hugoscottia / Tremolerasi m." handwritten by Knisch, ex coll. Bruch.

Notes: Córdoba was not mentioned in the original publication; moreover the specimen was labelled by Knisch after the publication date. The lectot, is at MLP, fide Fernández \& Cabrera (1996: 9). Lectot. and paralectot. designated by Fernández (1994: 40, f. 5-9). Recorded as Enochrus (Hugoscoltia) Tremolerasi by Orchymont (1943: 63).

\section{BIBLIOGRAPHY}

Archangelsky, M. 1990. A new species of the genus Phaenonotum Sharp, 1882 (Coleoptera, Hydrophilidae, Sphaeridinae) from Venezuela. Aquat. Ins. 11 (4), 1989: 217-220, f. 1-3.

- 1991. El género Phaenonotum Sharp en la Argentina. I. Redescripción de Phaenonotum regimbarti Bruch, 1915 y Ph. argentinense Bruch, 1915 (Coleoptera, Hydrophilidae, Sphaeridinae). Revta. Soc: entom. argent. 49, 1990: 157-164, f. 1-8.

- 1992. El género Phaenonotum Sharp en la Argentina (Coleoptera: Hydrophilidae). IE. Reubicación de Hydroglobus puncticollis Bruch, 1915, dentro del género Phaenonotum. Revta. Soc. 
entom. argent. 51: 47-51, f. 1-3.

Bachmann, A.O. 1966. El grupo de Stethoxus insularis (Coleoptera, Hydrophilidae). Revta. Soc. entom. argent. 28: 9-16, $1 \mathrm{pl}$.

- 1969a. Notas sobre Hydrophilini de Venezuela (Coleoptera, Hydrophilidae). Mem. Soc. Cienc. nat. La Salle, Caracas 28 (81): 281-300, f. 1-17.

- 1969b. Tropisternus (Pristoternus) gaeat una nueva especie de la República Argentina (Coleoptera Hydrophilidae), Revta. Soc. entom. argent. 31: 9396, f. $1-6$.

-.- 1970. Dos especies nuevas del género Tropisternus Solier (Coleoptera, Hydrophilidae). Revta. Soc. entom. argent. $32: 57-61$, f. 1-8.

- 1988. Las especies americanas de Hydrobiomorpha (Coleoptera, Hydrophilidae). Opera lilloana, Tucumán 36: 1-63, pl. i-xii.

- 1993. Nota nomenclatorial sobre Tropisternus (Col. Hydrophilidae). Revta. Soc. entom. argent. 50, 1991: 50,72 .

Berg, C. 1885. Quindecim Coleoptera nova faunae Reipublicae Argentinae. Anls. Soc. cient. argent. 19: 219-235.

Brèthes, J. 1922. Descripción de varios coleópteros de Buenos Aires. Anls. Soc. cient, argent. 94:263-307, $9 \mathrm{f}$.

Bruch, C. 1915. Nuevas especies de coleópteros hidrofilidos. Revta. Mus. La Plata 19 (2) (2a Ser. 6): $447-470$, f. $1-21$.

Fernández de Morras, L.A. 1981. Dos especies nuevas del género Helochares (Coleoptera Hydrophilidae). Limnobios, La Plata 2 (3): 189-192, f. 1-8.

Fernández, L.A. $1982 \mathrm{a}$. Notas sobre el género Helochares (Insecta, Coleoptera, Hydrophilidae). Neotrópica, La Plata 28 (79): 31-40, f. t-5.

- 1982b. Cinco especies nuevas del género Helochares (Coleoptera: Hydrophilidae). Physis, B. Aires, Secc. B 40 (99): 85-90, f. 1-9.

- 1983. Helochares (Sindolus) talarum sp. nov., redescripción de Helochares (Helochares) pallipes (Brullé) y descripción de sus estados preimaginales (Coleoptera Hydrophilidae). Limnobios, La Plata 2 (6): 439-449, pl. i, ii.

- 1988. Consideraciones sobre el género Chasmogenus Sharp y descripción de Chasmogenus sapucay sp. nov. (Coleoptera Mydrophilidae). Neotrópica, La Plata 32 (88), 1986: 189-193, pl. 1: f. 1-9.

- 1989a. Notas sobre el género Helochares. II. (Coleoptera: Hydrophilidae). Descripción de dos especies nuevas neotropicales. Clave para determinar las especies argentinas y de areas vecinas. Revta. Soc. entom. argent. 45, 1986: 145151, f. 1-24.

- 1989b. Las especies de Enochrus descriptas por Bruch. Redescripciones basadas sobre el material típico. Revta. Soc. entom. argent. 46, 1987: 121-128, f. $1-14$.

- 1990. Contribución al conocimiento del género Enochrus Thomson. 1. (Coleoptera: Hydrophilidae). Physis, B. Aires, Secc. B 46 (111), 1988: 85-89, f. 1 16.

- 1994. Contribución al conocimiento del género Enochrus Thomson (Coleoptera: Hydrophilidae). II.
El subgénero Hugoscottia en la Argentina. Physis, B. Aires Secc. B 49 (116-117): 37-45, f. 1-24.

- 1997. Nuevos aportes para el conocimiento del género Enochrus Thomson (Coleoptera: Hydrophilidae). Physis, B. Aires Sece. B 53 (124125): 21-29, f. 1-26.

Fernández, L.A. \& A.O. Bachmann. 1981. Nota sobre Hydrophilidae neotropicales (Coleoptera). I. Revta. Soc. entom. argent. 39 (3-4): 271-277, f. 1-18.

- 1987. Revisión del género Helobata Bergroth (Coleoptera, Hydrophilidae). Revta. Soc. entom. argent. 44 (2), 1985: 148-159, f. 1-19.

Fernández, L.A., A.O. Bachmann \& M. Archangelsky. 2001. Nota sobre Hydrophilidae neotropicales (Coleoptera). II. Nuevos taxiones de Tropisternus. Revta. Soc. entom. argent. 59, 2000: 185-197, f. 138.

Fernández, L.A. \& N.C. Cabrera. 1996. Los ejemplares tipo de Dytiscoidea, Haliploidea, Gyrinoidea e Hydrophiloidea depositados en la colección del Museo de La Plata. Revta. Mus. La Plata, Ser técn. $y$ didáct. (24): 1-10.

Hansen, M. 1989. A new name for the water beetle subgenus Tropisternus s. str. (sensu Orchymont, 1919) and lectotype designation of Hydrophilus collaris Fabricius, 1775. Steenstrupia, Köbenhavn $15(2): 53-56$.

- 1991. The Hydrophiloid beetles. Phylogeny, classification and a revision of the genera (Coleoptera, Hydrophiloidea). Biol. Skr. kong. danske Videnskab. Selsk. 40: 1- 367, f. 1-340, tab. a-g.

International Commission on Zoological Nomenclature. 1999. International Code of Zoological Nomenclature, $4^{\text {th }}$ edit.: xxix $+306 \mathrm{p}$.

Jensen Haarup, A.C. 1910. New species of Coleoptera from West Argentina. Deutsche entom. Zeitschr. Berlin 1910: 541-554.

Knisch, A. 1921a. Die exotischen Hydrophiliden des Deutschen Entomologischen Museums (Col.). Arch. Naturgesch. Berlin A 85 (8), 1919: 55-88.

- 1921b. Hydrophiliden aus Matto Grosso (Erster Teil). Arch. Naturgesch. Berlin A 87 (6), 1920: 124.

- 1922a. Hydrophiliden-Studien (Op. 10). Arch. Naturgesch. Berlin A 88 (5): 87-126.

- 1922b. Hugoscottia eine neue Helocharengattung (Col. Hydrophilidae Op. 12), Entom. Anz. Wien 2: 89-91, 103-105.

- 1924a. Neue neotropische Palpicornier (Col. Hydrophilidae - Op. 16). Wiener entom. Zeitg. 41 (4-10): 114-140.

- 1924 b. Hydrophilidae. In Schenkling, S. (Ed.) Coleopt. Catal. (79): 1-306.

- 1925. Coleoptera aus dem tropischen Amerika. Hydrophilidae (Palpicornia). Opus 18. Boll. Mus. Zool, Anat. compar. Torino, n. Ser. 39 (30): 1-12.

Makhan, D. 1992. Twelve new Hydrochus"species from South America (Coleoptera: Hydrophilidae), Phegea Amsterdam 20: 95-103.

- 1998. Three new genera and two new species of Hydrochidae from different parts of the World (Coleoptera). Annls. hist.rnat. Mus, nation. hungar. 
90: $139-150$, f. $1-30$.

Malcolm, S.E. 1981. A phylogeny and classification of the Sphaeridiinae (Coleoptera: Hydrophilidae). Unpubl. thesis Univ. Connecticut, USA: $\mathrm{xxi}+220$ p. (Not seen, cited after Hansen, 1991).

Mouchamps, R. 1959. Remarques concernant les genres Hydrobiomorpha Blackburn et Neohydrophilus Orchymont (Coléoptères Hydrophilides). Bull. Annls. Soc. entom. Belgique 95: 295-335, $49 \mathrm{f}$.

Oliva, A. 1982. El género Derallus Sharp en la Argentina (Coleoptera, Hydrophilidae). Reuta. Soc. entom. argent. 40, 1981: 285-296, pl. i, ii.

- 1983a. Las especies de Berosus descriptas por Brullé y por Jensen-Haarup de la Argentina (Coleoptera, Hydrophilidae). Revta. Soc. entom. argent. 42: 41-54, pl. i, ii.

- 1983b. Derallus de la cuenca del Amazonas (Coleoptera, Hydrophilidae). Revta. Soc. entom. argent. 42: 343-351, pl. i, ii

- 1985. Designación de lectotipo de Berosus (Enoplurus) bruchianus Knisch y sinonimia $\operatorname{con} B$. (E.) valparadiseus Moroni (Coleoptera, Hidrophilidae [sic] ). Reuta. Soc. entom. argent. 43: 191-194, f. 1-15.

- 1987. El género Epimetopus Lacordaire en la Argentina (Coleoptera: Hydrophilidae). Revta. Soc. entom. argent. 44 (1): 1-14, pl. i: f. 1-16; pl. ii: f. 1-6.

- 1989. El género Berosus (Coleoptera: Hydrophilidae) en América del Sur. Revta. Mus. argent. Cienc. nat., Entom. 6 (4): 57-236.

- 1992. The species of Hydrochus (Coleoptera; Hydrophilidae) described from South America. Bull. Annls. Soc. roy. belge Entom. 128: 87-104.

- 1993. Nuevas especies sudamericanas del género Berosus (Coleoptera: Hydrophilidae). Revta. Soc. entom. argent. 51, 1992: 87-95, f. 1-95.

- 1994a. A revision of the genus Hemiosus Sharp, 1882 in South America (Coleoptera: Hydrophilidae). Bull. Annls. Soc. roy, belge Entom. 130: 267-303, f, 1-32.

- 1994b. Una nueva especie de Hemiosus Sharp (Coleoptera: Hydrophilidae) de Venezuela. Revta. Soc entom. argent. 53: 75-77, f. 1-9.

- 1995a. A new species of Berosus Leach from northwesternArgentina (Coleoptera, Hydrophilidae). Bull. Annls. Soc. roy. belge Entom. 131: 57 60, f. 1-7.

- 1995b. Sur quelques Berosini (Coleoptera: Hydrophilidae) de la collection Lizer y Trelles. Bull. Annls. Soc. roy. belge Entom. 131: 75-77.

- 1996a. First mention of the genera Beralitra Orchymont, 1919 (with a new species) and Oocyclus Sharp, 1882 (Coleoptera: Hydrophilidae: Laccobiini) from Argentina. Bull. Annls. Soc. roy. belge Entom. 132 (1): 35-43, f, 1-5.

- 1996b. The genus Hydrochus Leach (Coleoptera; Hydrophiloidea; Hydrochidae) in South America, with special reference to Argentina. Bull. Annls.
Soc, roy. belge Entom. 132 (3): 301-341, f. 1-31.

- 1999. A new species of Berosus Leach from southeastern Brazil (Coleoptera: Hydrophilidae). Bull. Annls. Soc roy. belge Entom. 134, 1998: 239246 , f. $1-18$.

- 2000. Hydrochus synonymies. Latissimus, Balfour Browne Club (12): 14.

d'Orchymont, A. 1919. Contribution à l'étude des sousfamilles des Sphaeridiinae et des Hydrophilinae (Coleoptera, Hydrophilidae). Annls. Soc. entom. France 88: 105-168, f. 1-8.

- 1926. Contribution à l'étude des Hydrophilides. VI. Bull. Annls. Soc. entom. Belgique 66: 201-248.

- 1936. Scientific results of the Vernay-Lang Kalahari Expedition, March to September, 1930. Hydrophilidae. Annls. Transvaal Mus. 17 (2): 109-116, pl. i.

- 1937. Contribution a l'étude des Palpicornia. IX. Bull. Annls. Soc. entom. Belgique 77: 213-255.

- 1938. Contribution à l'étude des Palpicornia. XI. Bull. Annls. Soc. entom. Belgique 78: 261-270.

- 1941. Palpicornia (Coleoptera). Notes diverses et espèces nouvelles. I. Bull. Mus. roy. Hist, nat. Belgique 17 (1): 1-23.

- 1942. Contribution à l'étude de la tribu Hydrobinin Bedel, spécialement de sa sous-tribu Hydrobiae (Palpicornia - Hydrophilidae). Mém. Mus. roy. Hist. nat. Belgique $2^{e}$ Sér. (24): 1-68.

- 1943. Faune du Nord-Est Brésilien (Récoltes du Dr. o. Schubart). Palpicornia. Mém. Mus. roy. Hist. nat. Belgique 2e Sér. (28): 1-85.

Perkins, P.D. 1980. Aquatic beetles of the family Hydraenidae in the Western Hemisphere: classification, biogeography and inferred phylogeny (Insecta: Coleoptera). Quaest. entom., Edmonton, Canada $16(3): 1-554,198 \mathrm{f}$.

Spangler, P.J. 1977. Three new Ecuadorian species of the aquatic beetle genus Chaetarthria (Coleoptera: Hydrophilidae), Proc. biol. Soc. Washington 90 (3): 566-578, f. 1-15.

- 1986. A new genus and species of water scavenger beetle, Guyanobius adocetus, from Guyana and its larva (Coleoptera: Hydrophilidae: Hydrobiinae). Proc. entom. Soc. Washington 88 (3): 585-594, f. 124.

- 1990. A new species of water scavenger beetle, Guyanobius simmonsorum, from Brazil (Coleoptera: Hydrophilidae). Proc. entom. Soc. Washington 92 (3): 411-415, f. 1-13.

Wooldridge, D.P. 1973. New Paracymus from South America (Coleoptera: Hydrophilidae). J. Kansas entom. Soc. 46 (1): 116-123, f. $1-16$.

Recibido: $30-\mathrm{X}-2000$ Aceptado: 9-IV-2001 